\title{
Über allen Gipfeln ist Ruh
}

\author{
Zum Verhältnis von Friedensforschung und IB
}

\begin{abstract}
Zwischen Friedensforschung und IB besteht kein Schisma, im Gegenteil, es zeigt sich eine vielfältige personale Verflechtung, inhaltliche Gemeinsamkeiten und die geteilte Herausforderung der Veränderungen im Wissenschaftssektor. Auch zu den Sicherheits- und strategischen Studien sind die Grenzen eher fließend. Friedensforschung unterscheidet sich durch die Interdisziplinarität (mit Politikwissenschaft als Leitwissenschaft) und die klare normative Orientierung, die eigene Forschung auf den Zweck der Gewaltminderung auszurichten. Auch die IB mag dieses Ziel verfolgen, es ist ihr aber nicht »qua definition « eingeschrieben. Friedensforschung hat im Übrigen wie ihre Verwandten auch praktische Interessen und ist in der Medienarbeit und der Politikberatung aktiv. Für die künftige Forschung sind vor allem disziplinübergreifende »Grenzgänge« von Interesse.
\end{abstract}

\section{Einleitung}

In den folgenden Ausführungen halte ich mich an die Fragen, welche die ZIB-Herausgeber den Podiumsteilnehmern für die Diskussion auf der Sektionstagung in München und später für die Verschriftlichung gestellt haben. Ich lege zunächst meine Auffassung dar, dass von einem gegenwärtigen Auseinanderdriften von Friedensforschung ${ }^{1}$ und IB - über die seit jeher bestehenden Unterschiede hinaus nicht die Rede sein kann (Abschnitt 2). Sodann versuche ich, die Gemeinsamkeiten und Unterschiede zwischen beiden systematisch darzulegen (Abschnitt 3). Die Gretchenfrage nach der Normativität der Friedensforschung ist Gegenstand des vierten Teils. Das Verhältnis von Grundlagenforschung/Theoriearbeit und Praxis folgt im fünften Abschnitt, wobei ich auch auf die Zweige »Sicherheitsstudien« und »strategische Studien« kurz eingehe. Desiderate künftiger Forschung bilden den sechsten und letzten Block. In den Abschnitten 2, 3 und 4 schreibe ich als jemand, der als Forscher seit den siebziger Jahren in der Friedensforschung tätig ist, aber auch Beiträge zur Entwicklung der Internationalen Beziehungen geleistet hat, so-

1 Meine Entscheidung für den Terminus »Friedensforschung « ist pragmatisch. Frieden und Konflikt sind zwei Seiten einer Medaille - man kann über den einen nicht sprechen, ohne den anderen mitzuführen. Man könnte also mit gleicher Berechtigung »Friedens- und Konfliktforschung « sagen (wie ja auch der Name meines Instituts zeigt). Friedensforschung ist aber kürzer, nimmt weniger Platz weg, man verbraucht weniger Papier und weniger Bäume müssen gefällt werden. »Friedenswissenschaft« ist im Inland gelegentlich mit der Konnotation eines normativen Kampfbegriffs verbunden, im englischsprachigen Umfeld als »peace science« mit einer szientistischen Vorstellung von Sozialwissenschaft, die nicht die meine ist. Daher belasse ich es bei »Friedensforschung«. 
wohl in der Theoriebildung als auch institutionell, u.a. als Mitglied des Sektionsvorstands und Mitgründer der ZIB in der ersten Hälfte der neunziger Jahre. In den Abschnitten 5 und 6 stütze ich mich stark auf meine Perspektive als Leiter des Friedensforschungsinstituts Hessische Stiftung Friedens- und Konfliktforschung (HSFK).

\section{Friedensforschung und IB - Scheidung auf Münchnerisch?}

\subsection{Signale der Spaltung?}

Die Ausgangsfrage des Symposiums lautete, ob Friedensforschung und IB auseinanderdriften. Meiner Meinung nach kann man hier getrost Entwarnung geben. Ich habe schlimmere Schismen erlebt: Vor allem die helle Empörung, mit der sich in den siebziger Jahren die Honoratioren der deutschen IB aus der Arbeitsgemeinschaft Friedens- und Konfliktforschung zurückzogen. Freilich waren damals diese Auseinandersetzungen keineswegs auf die Friedensforschung beschränkt, sie spielten sich mit gleicher Wucht innerhalb der Sektion Internationale Beziehungen der Deutschen Vereinigung für Politische Wissenschaft (DVPW) ab und führten schnurstracks zur Gründung der Deutschen Gesellschaft für Politikwissenschaft. Letztlich zählte das Ganze zu den Nachwehen der 68er Revolte, stellte eine Konfrontation zwischen Mitte-Links und ganz Links dar (für eine Momentaufnahme vgl. Senghaas 1971 mit dem legendären Artikel von Lars Dencik über revolutionäre Gewalt als Friedensstrategie). Mit dem Älterwerden der ganz Linken wurden die Beziehungen milder; Ende der Achtziger, zu Beginn der neunziger Jahre konstituierte sich nicht nur die Sektion Internationale Beziehungen der DVPW neu (unter maßgeblicher Beteiligung von Friedensforschern wie Volker Rittberger, Klaus Dieter Wolf oder auch mir), sondern »HSFKler« arbeiteten ziemlich dauerhaft im Vorstand der Arbeitsgemeinschaft für Friedens- und Konfliktforschung (AFK) mit: Das Schisma war vorbei.

Ganz sicher ist die Gründung einer neuen Zeitschrift für Friedensforschung mit peer review-Verfahren nicht als Zeichen eines neuen Schismas zu verstehen. Man muss das gelassen sehen. Als die ZIB 1994 aus der Taufe gehoben wurde, gab es bereits drei etablierte Zeitschriften der Friedensforschung: Die von der HSFK herausgegebenen »Friedensanalysen«, »Sicherheit und Frieden« des Instituts für Friedensforschung und Sicherheitspolitik (ISFH) und die altehrwürdige »Friedenswarte«. Kein Mensch hat die Gründung der ZIB - der ersten deutschen politikwissenschaftlichen Zeitschrift mit Gutachterverfahren - als Attacke auf die Friedensforschung verstanden. Das wäre auch schön unsinnig gewesen, denn die »Gründerväter«, Reinhard Meyers, Klaus Dieter Wolf und ich, waren allesamt in der Friedensforschung verankert. Die Friedensanalysen wurden wenig später eingestellt, aber das hatte mit der ZIB rein gar nichts zu tun. Die heutigen Gründer der Zeitschrift für Friedens- und Konfliktforschung (ZeFKo), Thorsten Bonacker, Tanja Brühl und Christoph Weller sind sämtlich ausgewiesene »IBler«, der Letztere 
war zudem Gründungsredakteur der ZIB und hat deren Format ganz maßgeblich geprägt.

Auch die neuen Masterstudiengänge für Friedensforschung sind kein Zeichen einer Friedensforschungs-Sezession. Sie sind dem Spezialisierungszwang geschuldet, den der Bologna-Prozess unabhängig von allen »IBlern « und Friedensforscherinnen nun einmal ausübt. Die Fachbereiche sind genötigt, attraktive Angebote für intelligente Studierende zu machen. Friedensforschung hat für engagierte junge Menschen eine unverkennbare Anziehung. Ein gemeinsamer Masterstudiengang der Universitäten Frankfurt und Darmstadt, der in enger Zusammenarbeit mit der HSFK entwickelt wurde und in dessen Rahmen Mitarbeiter/innen der HSFK lehren, heißt im Übrigen »Internationale Studien/Friedens- und Konfliktforschung» - es geht also schön harmonisch zu.

\subsection{Professionalisierung, Evaluierung und ihre dunklen Seiten}

Die Gründung einer neuen Zeitschrift lässt sich, ebenso wie die Ausbreitung neuer Masterstudiengänge, als Teil der Professionalisierung der Disziplin verstehen, die mit einem heftigen Wettbewerb verbunden ist, welcher nicht nur positive Auswirkungen hat. Die Professionalisierung, die seit mehr als einem Jahrzehnt nicht nur von den Instituten und Universitäten, sondern auch von der Spitze der AFK gezielt voran getrieben wird, schlägt sich in steigender methodologischer, empirischer und theoretischer Qualität der Projekte und Publikationen nieder. Sie hat leider auch eine negative Seite: Institutionen sowie individuelle Wissenschaftlerinnen und Wissenschaftler sind einem ständigen, in seiner Ubiquität mittlerweile manischen und nicht mehr produktiven Evaluierungszwang unterworfen, der z.T. perverse Anreize für die wissenschaftliche Tätigkeit setzt. Die hohe (Über-)Bewertung des Zeitschriftenaufsatzes mit Gutachterverfahren durch Berufungskommissionen und andere Evaluationsgremien schafft zwangsläufig - bei einer noch steigenden Zahl von wissenschaftlich Tätigen - den Bedarf nach genügenden »Outlets«, woraus sich die ständig wachsende Zahl der Zeitschriften erklärt. Ob das eine weitere Qualitätszeitschrift im deutschen Sprachraum rechtfertigt, mag man diskutieren - der Markterfolg der Zeitschrift für Friedens- und Konfliktforschung wird diese Frage beantworten. Die dunkle Seite der Angelegenheit: Der Evaluierungszwang, von dessen erfolgreichem Durchlauf die Berufschancen der Nachwuchswissenschaftlerinnen und Nachwuchswissenschaftler und die leistungsabhängigen Gehaltsbestandteile der Etablierten abhängen, tendieren dazu, Substanz und normative Orientierung durch die Ausrichtung auf das bloße Zählen der eigenen Publikationen und die Anpassung an die höchstbewerteten und größten Publikationsmärkte - die englischsprachigen und vor allem den amerikanischen - zu verdrängen. In den letztgenannten existiert eine wissenschaftstheoretische Hegemonie zugunsten multivariater large$n$-Studien und möglichst formalisierter rational-choice-orientierte Aufsätze mit der tendenziellen Folge einer Entpluralisierung der Wissenschaftslandschaft. Nichts gegen diese beiden Ansätze, aber wenn am Ende nur sie übrig bleiben sollten, hätten 
wir eine amputierte Sozialwissenschaft, basierend auf zweifelhaften Kausalitätsannahmen und einer verkürzten anthropologischen Grundlage - gerade für die Friedensforschung keine attraktiven Aussichten!

Das hier Ausgeführte trifft IB und Friedensforschung in gleichem Maße. Beide sitzen in diesem Sinne im selben Boot. Von einem Schisma vermag ich nichts zu entdecken. Wir können uns also in Ruhe der Aufgabe zuwenden, Gemeinsamkeiten und Unterschiede der beiden Forschungsrichtungen zu besehen.

\section{Friedensforschung und IB: Fachzugehörigkeit versus Interdisziplinarität}

Die Friedensforschung blickt auf eine bestimmte soziale Relation - den Frieden -, das aber aus allen denkbaren Perspektiven. Weil es sich um eine soziale Relation handelt, stehen die Sozialwissenschaften im Zentrum. Da es überwiegend - aber nicht ausschließlich - politische Verhältnisse und politisches Handelns sind, die den Frieden gefährden, zerstören, wiederherstellen oder stabilisieren (und auch z.B. ökonomische Kriegsursachen erst in ihrer politischen Vermittlung zur Gefahr für den Frieden werden), hat die Politikwissenschaft den Status einer Leitwissenschaft der Friedensforschung erlangt. Außer der auf grenzüberschreitende Konflikte spezialisierten IB braucht die Friedensforschung auch die Instrumente zur Analyse der inneren Verhältnisse von Staaten und ihrem äußeren Konfliktverhalten, braucht Einsichten in innere Konflikte, in die Dynamik transnationaler Prozesse. Der Konflikt gehört übrigens zum Frieden wie ein siamesischer Zwilling. Friedensforschung ist zwangsläufig immer auch Konfliktforschung. Zum einen geht es um das Risiko, dass Konflikte so eskalieren können, dass sie den Frieden stören - wenn man den Frieden erhalten will, muss man also den Konflikt verstehen. Zum anderen ist eine wesentliche Voraussetzung friedlicher Beziehungen, allfällige Konflikte ohne das Mittel der Gewalt austragen zu können, denn Frieden ist nicht gleichbedeutend mit dem Ende aller Konflikte - das wäre eher Friedhofsruhe -, sondern mit deren Austrag ohne den Rückgriff auf Gewalt.

Als soziale Relation, deren Bestand ebenso wie ihre Gefährdungen komplex und voraussetzungsreich sind, tragen alle möglichen Faktoren zum Frieden oder seiner Negation bei, die in den Sachbereich anderer Wissenschaften fallen; Friedensforschung ist daher notwendigerweise interdisziplinär. In der Hessischen Stiftung Friedens- und Konfliktforschung sind neben der Politikwissenschaft die Philosophie, die Kernphysik, die Soziologie, die Ethnologie, die Kulturwissenschaft, die Religionswissenschaft, das Völkerrecht und die Volkswirtschaft vertreten. Auch Historiker, Pädagogen und Psychologen haben schon an der HSFK gearbeitet. Während meiner Mitgliedschaft im Stiftungsrat der Deutschen Stiftung Friedensforschung haben wir über die genannten Fächer hinaus auch Anträge aus der Biologie, der Informatik, der Theologie, der Umweltwissenschaft und der Linguistik behandelt. All diese Fächer bieten Zugänge zum zentralen Thema der Friedensgefährdung und Friedensgestaltung. Friedensforschung ist daher kein Teilfach ihres Leitfachs Politikwissenschaft, sondern ihrem Wesen nach über eine Vielzahl wissenschaftlicher 
Fächer verteilt. In diesem Sinne ist die Friedensforschung keine eigene Disziplin, sondern ein auf eine zentrale Thematik gerichteter Forschungsverbund.

Die Internationalen Beziehungen sind gleichfalls durch ihren Gegenstand gekennzeichnet. Freilich ist dieser zugleich fachlich definiert, als politische Beziehungen. Die IB waren daher seit den Anfängen der Politikwissenschaft in Deutschland deren Teildisziplin. Freilich entgeht sie damit ihrem Schicksal nicht, Probleme mit der disziplinären Abgrenzung zu haben. So wie die Friedensforschung in andere Teildisziplinen der politischen Wissenschaften ausgreift, in die Institutionenlehre, die vergleichende Politikwissenschaft, die politische Soziologie, die politische Ökonomie und die politische Theorie, so kommen auch die IB nicht ohne Grenzüberschreitungen in diese Richtungen aus. Eine ihrer (mit der Friedensforschung geteilten) prominentesten Theorien, der demokratische Frieden, ist nichts anderes als vergleichende Institutionenlehre plus politische Theorie - durch den Rückgriff auf Kant. Und mehr noch: Auch die IB brauchen den Blick auf das Völkerrecht (aus dem sie ja einmal entstanden sind), die Geschichtswissenschaft (man sehe sich große IB-Monographien wie die von Kenneth Waltz, Alexander Wendt, Ned Lebow oder Daniel Deudney an, die alle eine beträchtliche historische Dimension aufweisen, von Hans Morgenthau ganz zu schweigen). Auch eine gewisse wirtschaftswissenschaftliche Kompetenz tut den IB gut, d.h. die Verzahnung mit der Internationalen Politischen Ökonomie (IPÖ), um mit den notorischen Verflechtungen von Wirtschafts-, Macht- und Konfliktbeziehungen zu Rande zu kommen. Wer - außer vielleicht sehr sektiererischen Neo-Realisten - hält die IB noch für eine Disziplin mit scharfen Grenzen? Aus diesem Grund haben Gunther Hellmann und ich (2004) in unserer gemeinsamen Zeit als verantwortliche Herausgeber der ZIB die Zeitschrift für »Grenzgänge« geöffnet, für den kühnen Drahtseilakt zwischen den Disziplinen und Teildisziplinen. Und deshalb ist es auch Sabotage am Erkenntnisfortschritt unserer Wissenschaft, wenn Berufungskommissionen immer wieder Bewerberinnen und Bewerber ablehnen, die diesen Grenzgang unternehmen, mit der provinziellen Begründung, sie hätten kein »disziplinäres Profil«.

Der Unterschied zwischen dem »Forschungsverbund « Friedensforschung und der »Teildisziplin« IB schrumpft also zu dem Befund zusammen, dass Ersterer eine interdisziplinäre Veranstaltung mit Politikwissenschaft als Leitwissenschaft, Letztere eine politikwissenschaftliche Teildisziplin ist, die auf den Rückgriff auf andere (Teil-)Disziplinen angewiesen ist. Kein Wunder, dass diese beiden genetisch ähnlichen Cousinen ganz gut miteinander auskommen!

\section{Wie hältst Du's mit dem Normativen?}

\subsection{Von der Normativität der Friedensforschung}

Friedensforschung ist eine Wissenschaft mit normativem Auftrag. Ich nähere mich diesem Punkt auf dem Umweg über eine Definition. Ich neige dazu, den Frieden minimalistisch zu definieren, nämlich ausschließlich im Bezug auf physische Ge- 
walt (Müller 2003). Die maßlose Aufblähung des Friedens- wie des Gewaltbegriffs über die Konstrukte »positiver Frieden« und »strukturelle Gewalt« haben sich weder in der Wissenschaft noch in der gelegentlichen verballhornten Übernahme in die politische Sprache bewährt. Wie bei allen Begriffen bekommt auch diesem die Entgrenzung nicht gut, wenn es um Theoriebildung und um Empirie geht. Die Gallerte grenzfreier Begriffe ist nicht zu packen. Hinzu kommt die Missbrauchsmöglichkeit eines derart »leeren Signifikanten« für politische und diskriminierende Vorhaben. Man lese, wie der späte Johan Galtung (z.B. 1997) seinen Antiamerikanismus im virtuosen Spiel mit dem entgrenzten Gewaltbegriff auslebt. So lässt sich große Polemik machen, aber keine gute Wissenschaft. Ebenso wenig ist es hilfreich, andere soziale Beziehungsbegriffe wie Gleichheit oder Gerechtigkeit zu Definitionsbestandteilen des Friedens zu machen - alle verlieren dabei, nämlich an Schärfe und damit an Einsatzfähigkeit. Man setzt den Begriff der Gerechtigkeit ja nicht herab, wenn man ihn als eigene soziale Beziehung begreift, die erstrebenswert ist (jedenfalls wenn sich die Akteure darauf einigen können, was damit gemeint sei) und mit der sozialen Beziehung Frieden in einem engen Verhältnis steht, aber eben weder dasselbe noch Teil davon ist; vor allem die höchst ambivalente Relation zwischen Frieden und Gerechtigkeit wird von diesen Vereinnahmungen zugekleistert (Müller 2011).

Aber selbst in der minimalistischen Version ist Frieden als die Annäherung an eine ideale Ordnung zu verstehen, eben weil die Minderung von inner- und zwischenstaatlicher Gewalt in Richtung Null derart voraussetzungsreich ist, dass eine gedachte Friedensordnung stets eine kontrafaktische Idealisierung darstellt. Vorstellungen dieser Art stehen immer in Gefahr, ideologisch zu werden, der man sich bewusst sein muss. Die Minderung physischer Gewalt ist in jedem Fall ein sinnvolles pragmatisches Ziel, das der wissenschaftlichen Arbeit als normative Richtschnur dienen kann.

Damit wäre der erste normative Pfeiler der Friedensforschung errichtet. Der zweite besteht in der Handlungssystem-Perspektive. Frieden ist nicht die Sache eines singulären Akteurs, sondern eben die Beziehung zwischen zwei oder mehreren Akteuren. Frieden kann nicht für einen von ihnen bestehen, für die anderen aber nicht. Die Friedensforschung bezweckt daher immer, zur Befriedung eines Handlungssystems (Familie, Gruppe, Gesellschaft, Region etc.) beizutragen. Das trägt ihr leicht den Ruf der »fünften Kolonne« ein, wie ich selbst zu Zeiten des Kalten Krieges noch schmerzlich erfahren habe, weil wir »pflichtgemäß« eben auch die Perzeptionen und Sicherheitsbedürfnisse der »anderen Seite« untersuchten.

Der dritte normative Pfeiler ist die Einnahme der Opferperspektive. Um das Missverständnis auszuschließen, dass damit dem herkömmlichen bias von Gewaltakteuren, sie seien das Opfer der teuflischen Handlungen des jeweiligen Feindes, das Einfallstor in die Friedensforschung geöffnet werden könne: »Opfer« meint hier den zivilen »Kollateralschaden«, den jegliches organisierte Gewalthandeln verursacht. Politik rollt häufig über die Menschen wie ein gewaltiger juggernaut, ob sie böse oder gut gemeint ist. Im Besonderen gilt das, wenn Politik zur Waffe greift: Auch die humanitärste Intervention erzeugt ihre zivilen Opfer, die mit den 
Gründen, welche zu der militärischen Operation geführt haben, in keiner Weise schuldhaft verbunden sind. Im Grenzfall kann das das geringere Übel sein - dass es dennoch auch diese üble Seite ethisch motivierter Politik geben kann, ist analytisch nicht aus dem Blick zu verlieren; auch das gehört zur normativen Aufgabe der Friedensforschung. Ein Frieden, der die Opfer vergisst, ist eine faule Angelegenheit, und das gilt mutatis mutandis auch für seine Erforschung.

All das macht wissenschaftliches Arbeiten nicht unmöglich. Für uns politikwissenschaftliche Friedensforscher ist das gesamte methodologische Spektrum des Faches einsatzfähig. Von der large- $n$-Studie bis zum Fallvergleich, von der deduktiven Hypothesenableitung bis zur induktiven grounded theory lässt sich alles nutzbar machen, wenn es dem jeweiligen Forschungszweck dienlich ist. Dass eine Wissenschaft, die sich zu einem normativen Ziel bekennt, nicht mehr wissenschaftlich vorgehen könne, ist ein nicht sehr geistreiches Märchen. Wäre das so, dann könnte auch die Medizin keine Wissenschaft sein, denn die steht unter dem hippokratischen Eid, und etwas Normativeres gibt es nicht.

Das Normative an der Friedensforschung, das alle drei »Pfeiler« gemeinsam haben, ist ihr Gerichtetsein auf ein normativ verstandenes Ziel, Frieden. Sie will dazu beitragen, dass mehr Frieden wird. Das ist für die »institutionalisierte« Friedensforschung übrigens auch institutionenrechtlich gar nicht zu vermeiden, weil es als Aufgabe in die Institutsverfassungen eingeschrieben ist. Und könnte es anders sein? Wie wäre denn eine Friedensforschung zu verstehen, die sich gegenüber dem Frieden neutral erklärte? Wäre sie dann auch bereit, Projekte für die Friedensstörung aufzulegen? Auftragsforschung für Aggressoren zu betreiben? Doch wohl kaum. Die Forschung für den Krieg wäre auch riskant, denn die Freiheitsstrafen, die $\S 80$ StGB auf die Vorbereitung des Angriffskrieges (zu denen auch Unterstützungshandlungen zu rechnen sind) setzt, sind saftig. Eine Friedensforschung, die sich für nicht-normativ erklärt, hat, so vermute ich, über ihr eigenes Tun noch nicht gründlich genug nachgedacht, so wie viele Forschungsrichtungen, die sich für wertfrei halten, es de facto aber nicht sind.

\subsection{Sag mir, wo die Normen sind: IB, Sicherheits- und Strategieforschung}

Womit ich wieder bei den IB wäre. Die IB tragen die der Friedensforschung aufgebürdete normative Pflicht nicht in demselben Maße. Als normative Wissenschaft in die Welt getreten (man muss Morgenthau lesen, um zu verstehen, wie normativ!), hat sie - zumindest ihr hegemonialer US-amerikanischer Strang - seit der behavioralistischen Wende der sechziger Jahre danach gestrebt, sich dieser Bürde zu entledigen. $\mathrm{Zu}$ ihrer selbsterklärten Norm wurde die Produktion wissenschaftlicher Wahrheit über die Internationalen Beziehungen unter dem Banner des Positivismus. Auch sie trägt das Risiko, zur Ideologie zu verkommen - Tony Smith (2007) hat das am Beispiel des »demokratischen Friedens« eindrucksvoll demonstriert; ihre szientistische Attitüde macht es ihr indes noch schwerer, das Problem zu sehen, als es schon der Friedensforschung fällt. 
In den deutschen IB, wie in Europa überhaupt, hat der Szientismus nicht die hegemoniale Stelle erlangt wie in den Vereinigten Staaten, was im Übrigen auch der Dialogfähigkeit zwischen den verschiedenen Schulen eher gut tut. Ohne Weiteres lässt sich unterstellen, dass zwischen der normativen Orientierung der Friedensforscherinnen und Friedensforscher und denen des deutschen IB-mainstreams keine allzu scharfen Unterschiede bestehen. Auch »normale« »IBlerinnen« und »IBler« möchten mit ihrer Forschung $\mathrm{zu}$ friedlicheren und anderweitig besseren Beziehungen beitragen.

Nun gibt es im Rahmen der IB spezialisierte Teilgebiete, die unter den Namen »Sicherheitsstudien« und »strategische Studien« firmieren. Beide Richtungen sind in Deutschland nie so recht heimisch geworden, was mit der ausgesprochen zivilistischen Kultur der »Zivilmacht Deutschland« zusammenhing. Die beiden Begriffe hatten für viele den Ruch des Militärischen, wenn nicht Militaristischen. In den USA oder Großbritannien begegnete man - ohne die historische Last im Gepäck diesen Forschungsrichtungen mit weniger Vorbehalt; Security Studies und Strategic Studies zählen dort zum normalen Kanon von Forschung und Lehre. Dabei ist zu vermerken, dass in beiden eher eine nicht-akademische, politikanalytisch und praxeologisch ausgerichtete Richtung dominiert. Sie haben ihren Hauptsitz in außeruniversitären Think Tanks, vom International Institute for Strategic Studies (IISS) bis zum Research and Development (RAND); es finden sich aber auch in den Universitäten entsprechend denominierte Lehrstühle. Das Aufkommen von Critical Security Studies in den neunziger Jahren (Krause/Williams 1997; Booth 2007) verrät diese Tabulosigkeit, obgleich wir auf die Critical Strategic Studies noch warten. Ich knüpfe hier an den englischen Begriff der Security Studies an. Was in Deutschland und Europa neuerdings als Sicherheitsforschung gefördert wird, ist sehr viel weniger, als Security Studies bedeutet, nämlich überwiegend eine versteckte Subvention der deutschen Wehrindustrie, nachdem sich nun der Verteidigungshaushalt nicht allzu sehr steigern lässt, weil das gar zu unpopulär wäre. Der »Sicherheitsforschung« hat man dann noch ein sozialwissenschaftliches Feigenblatt verpasst, damit die obszöne Subventionsblöße nicht allzu lasziv zu Tage läge. Mit den klassischen Security Studies hat das Ganze wenig zu tun.

Von der normativen Ausrichtung der Security Studies her gibt es schon deutliche, aber auch nicht wirklich hermetische Unterschiede zur Friedensforschung. Den $\mathrm{Se}$ curity Studies haftet vom Begriff her noch eine gewisse Defensivität an, die sich mit der Einnahme der Gegenüber-Perspektive, des zweiten normativen Pfeilers der Friedensforschung, recht gut verträgt. Freilich tendieren Security Studies eher dazu, Sicherheitsbeziehungen mit dem Ziel zu untersuchen, die nationale Sicherheit zu stärken, nicht die Sicherheitsbeziehung des Handlungssystems mit all seinen Akteuren verbessern zu wollen. Das kann im Einzelfall auf dasselbe hinauslaufen, muss es aber nicht. Strategic Studies sind vom Begriff her nicht auf Defensive festgelegt, auch offensive Optionen können ihr Gegenstand und ihr Ziel sein. Man erinnere sich an die Studien von Colin Gray und Keith Payne zum »Sieg im Nuklearkrieg« (Gray/Payne 1980), die sicher in den Einzugsbereich der Strategic Studie fielen. Hier ist die normative Ausrichtung vollständig beliebig und ganz in das Er- 
messen des »strategischen Forschers« gestellt. Wie der Name schon sagt: Strategie ist ein Mittel zum Zweck. Der Instrumentalismus kann sich seine Zwecke beliebig suchen.

Summarisch lässt sich feststellen, dass die Grenzen zwischen den Ansätzen verschwommen sind. Klar ist Friedensforschung von strategischen Studien absetzbar, aber zwischen Friedensforschung und IB gibt es große, zwischen Friedensforschung und Security Studies noch nennenswerte Überlappungen. Die gibt es auch zwischen IB und Sicherheitsstudien, vielleicht eine kleine Teilmenge zwischen IB und strategischen Studien, und eine größere zwischen Letzteren und Sicherheitsstudien. $\mathrm{Ob}$ uns allerdings diese Begriffsarbeit jetzt wesentlich weiter gebracht hat, vermag ich nicht zu sagen.

\section{Theorie und Praxis}

Friedensforschung stellt an sich selbst den Anspruch, praktisch zu werden. Es geht nicht lediglich darum, über den Frieden, seine Störungen und seine Ursachen zu räsonieren, sondern auch darum, den Praktikern und Praktikerinnen Praxeologien zur Verfügung zu stellen, die zum Schutz und Verwirklichung des Friedens nützlich sein könnten. Manche Einrichtungen der Friedensforschung - etwa das BerghofForschungszentrum für konstruktive Konfliktbearbeitung - behandeln auch die Praxis selbst als Teil ihrer Kernkompetenz. Dieser praxeologische Zweck (in der HSFK-Satzung beispielsweise in dem luftigen Auftrag der »Verbreitung des Friedensgedankens « niedergelegt) hat die Friedensforschung nicht daran gehindert, intensive Grundlagenforschung zu betreiben. Als Mitglied der Leibniz-Gemeinschaft ist die HSFK gehalten, deren Leitmotiv »theoria cum praxi«, d.h. Grundlagenforschung und deren Umsetzung in handhabbare Praxeologien, parallel zu verfolgen. Keine leichte Aufgabe für das wissenschaftliche Personal, weil es eine erhebliche Spreizung der eigenen Kompetenzen, einen echten Spagat, verlangt, aber zugleich auch eine hoch interessante Herausforderung, auf mehreren Hochzeiten die dort verlangten Tanzschritte tun zu können, ohne als Dilettant unangenehm aufzufallen. Verlangt ist dabei nicht nur die Umsetzung des Erforschten in anwendbare Handlungsoptionen, sondern zugleich die Übersetzung aus der Sprache des Wissenschaftssystems in die der Praktiker und Praktikerinnen. Dazu bedarf es einer gewissen Flexibilität und Bereitschaft, das »Dolmetscherhandwerk« zu lernen sowie der Erfahrung. Die Transferleistung gleicht eher einer Kunstfertigkeit oder einer handwerklichen Leistung als dem Kanon guten wissenschaftlichen Arbeitens (Müller 2006).

Die HSFK betreibt den praxisorientierten Teil ihrer Arbeit in erster Linie durch Politikberatung, und hier am intensivsten in kontinuierlichen Kontakten zur Arbeitsebene des Auswärtigen Amtes (AA). HSFK-Mitglieder sitzen beispielsweise einer Arbeitsgruppe »Friedens- und Konfliktforschung« beim Planungsstab des AA sowie einer interministeriellen Beratungsgruppe zur zivilen Konfliktprävention vor und haben in den vergangenen zwanzig Jahren an Verhandlungsdelegationen in 
fünf verschiedenen Feldern der Rüstungskontrolle, Abrüstung und Nichtverbreitung mitgewirkt. Darüber hinaus erstrecken sich Beratungsleistungen auch auf andere Bundesministerien (Bundesministerium der Verteidigung, Bundesministerium für wirtschaftliche Zusammenarbeit und Entwicklung), auf den Deutschen Bundestag, Parteien, Nichtregierungsorganisationen, Unternehmen und die Hessische Landesregierung. Als Mitglied im Consortium for Non-Proliferation and Disarmament der Europäischen Union (EU) (gemeinsam mit der Pariser Fondation des Recherches Stratégiques, dem Londoner International Institute for Strategic Studies und dem Stockholm International Peace Research Institute, vgl. Rat der Europäischen Union 2010) berät die HSFK auch die Institutionen der EU sowie deren Mitgliedstaaten.

Insofern ist es nicht paradox, sondern folgerichtig, wenn die Friedensforschung, trotz ihrer erheblichen Ferne zu Strategic Studies, ebenfalls strategisch denkt; es geht eben auch darum, die vorhandenen Hindernisse auf dem Weg zum Frieden zu beseitigen und den Frieden bestmöglich in den Mentalitäten und Praktiken der Akteure und somit auch in den institutionellen Strukturen, in denen sie handeln, zu verankern. Am klarsten hat diese Forschungsrichtung der Friedensforschung ErnstOtto Czempiel (1998) in seinem Klassiker »Friedensstrategien« zum Ausdruck gebracht.

Für die IB ist (wie die explizite normative Orientierung) auch die praktische Orientierung eine optionale Angelegenheit. Dies hat zu einer (nicht unbedingt zuträglichen) Spaltung der Branche in überwiegend politikberatende Akteure (wie die Stiftung Wissenschaft und Politik oder die Deutsche Gesellschaft für Auswärtige Politik, aber auch manche Lehrstühle) und überwiegend akademisch/grundlagenforschungs-orientierte Akteure geführt, deren Maß an wechselseitiger Hochschätzung ebenso wie ihre Kommunikation zu wünschen übrig lassen. Natürlich verwenden auch die dem Elfenbeintrum entwöhnten Berater und Beraterinnen Theorie oder Theorieversatzstücke, weil das für die Beratung unerlässliche Ordnen und Interpretieren der »Fakten« theoriefrei nun einmal nicht möglich ist - dies allerdings oft implizit und unbewusst. Für die Beratenen bleibt somit im Dunkeln, was der eigentliche Hintergrund der erteilten Ratschläge ist - auch kein Optimum. Dasselbe gilt umgekehrt natürlich für eine Wissenschaft, die die Berührung mit der Praxis scheut. $^{2}$

Die Spaltung in eine eher akademische und eine eher praktisch orientierte Teildisziplin der Teildisziplin ist nun aber keine nationale Idiosynkrasie, sondern ent-

2 Ich hoffe, dass diese Ausführungen die durch keine empirische Untersuchung gedeckte Auffassung endgültig zu den Akten legen, die deutsche Friedensforschung sei eine praxisferne Veranstaltung, die sowohl von einem Symposiumsteilnehmer als auch von einem Gutachter vorgebracht wurden. Woher das kommt, weiß ich nicht. Tatsache ist, dass auch unser Hamburger Schwesterinstitut Institut für Friedensforschung und Sicherheitspolitik (IFSH), das Bonn International Center for Conversion (BICC), das Institut für Entwicklung und Frieden der Universität Duisburg-Essen und das Berliner Berghof Institut beständig im Kontakt mit der Praxis stehen und einen ununterbrochenen Fluss von Beratungsleistungen erbringen. Zusätzliche Anstrengungen, diesen Kommunikationsfluss zu erhalten und zu stärken, unternimmt seit ihrer Gründung mit gutem Erfolg die Deutsche Stiftung Friedensforschung. Wäre es für die Kritiker nicht geraten, erst einmal einen Blick in die empirischen Fakten zu tun, bevor sie sich mit derartigen Fehlurteilen hervorwagen? 
spricht durchaus dem Zustand in Großbritannien und den USA. Zwar gibt es in den USA das vielgerühmte Rotationssystem zwischen Wissenschaft und Administration, jedoch erfasst das nur einen Bruchteil der wissenschaftlichen Einrichtungen. Die große Mehrzahl der Wissenschaftlerinnen und Wissenschaftler kommen aus der Alma Mater nicht hinaus, und die Zahl der Koryphäen mit Verdiensten um die Theoriebildung wie Joseph Nye oder Stephen Krasner, die sich auch praktisch-politische Lorbeeren verdient haben, ist gering geblieben.

\section{Schwerpunkte künftiger Forschung}

Die Redaktion hat uns schließlich darum gebeten, unsere Auffassungen von den wünschenswerten künftigen Forschungsschwerpunkten der Friedensforschung kund zu tun. Als Leiter eines einschlägigen Instituts kann ich diese Frage verständlicherweise nicht unbefangen beantworten. Wir sind gehalten, in regelmäßigen Abständen ein kohärentes Forschungsprogramm aufzulegen, welches den größeren Teil der wissenschaftlichen Tätigkeit des Instituts zusammenbindet. Die von mir beschriebenen Schwerpunkte lehnen sich daher an die Thematik des gegenwärtigen Forschungsprogramms der HSFK an, das unter dem Titel »Just Peace Governance« läuft. ${ }^{3}$

\subsection{Ideationale Faktoren in der internationalen Politik}

Einen ersten Schwerpunkt sehe ich in der stärkeren Beschäftigung mit der normativen Seite von Politik. Dieser Ansatz ist zu unterscheiden von der oben diskutierten Normativität der Friedensforschung: Jene bestimmt den Zweck unseres Forschens, die Rolle von ideationalen Faktoren in der Politik hingegen ist ein spezifischer empirischer Gegenstand. Natürlich knüpft dieser Schwerpunkt an konstruktivistische Überlegungen an. Der IB-Konstruktivismus hat sich indes überwiegend mit der Herausbildung internationaler Normen und deren Wirkung auf der staatlichen Ebene befasst. Mir geht es hier um die intrinsische Wert- und Normengebundenheit von Politik auch jenseits internationaler Normensysteme. Dass ideationale Faktoren wenigstens gleichberechtigt mit »Interessen « das Handeln von Regierungen bestimmen, war den Klassikern noch völlig klar - Morgenthau (1948) etwa plädierte leidenschaftlich für die Stärkung des Interessenfaktors in der Außen- und Sicherheitspolitik im Vergleich zur Moral, weil er um die Sicherheit der demokratischen Länder durch die überstarke Unterstellung gleicher moralischer Motive auf der Gegenseite (appeasement-Problem) oder durch aggressiv gewendete moralische Ansprüche (Kreuzzugs-Problem) fürchtete.

3 Ausgangsüberlegungen finden sich unter verschiedenen Perspektiven in Baumgart-Ochse et al. (2011). 
Die Virulenz ideationaler Faktoren ist seit mehr als einem Jahrzehnt durch die zunehmende politische Bedeutung religiösen Denkens und Fühlens sichtbar geworden, erschöpft sich darin aber nicht. Das HSFK-Forschungsprogramm »Just Peace Governance« hat mit »Gerechtigkeit« ein ideationales Konzept ausgewählt, dessen inhaltliche Füllung sich sowohl aus religiösen als auch aus säkularen Quellen speist. Für die Untersuchung der Internationalen Beziehungen ist es nur höchst selektiv berücksichtigt worden (z.B. Welch 1993; Albin 2001; Mayer 2005). Folgt man indes diesen Pionierarbeiten, so üben Gerechtigkeitsansprüche eine erhebliche Trieb- und Sprengkraft gegenüber politischen Konflikten und normativen Ordnungen aus, die es weiter zu untersuchen gilt.

\subsection{Kulturelle Vielfalt und Transnationalismus}

In diesem Zusammenhang werden sofort die vielfältigen Zugänge zur Gerechtigkeitsproblematik wichtig. Sie variieren sowohl innerhalb kultureller Systeme als auch zwischen ihnen. Dadurch entstehen Konfliktfronten, die es zu überwinden gilt, sollen einvernehmliche Regelungen (»Governance«) für virulente Probleme in den internationalen Beziehungen gelingen. In diesem Zusammenhang spielt das steigende Gewicht transnationaler Akteure, Strukturen und Prozesse eine maßgebliche Rolle, durch die sich ursprünglich lokal bzw. regional gebundene Wertsysteme globalisieren, was sowohl ihre Konfliktträchtigkeit steigern, als auch zur Bearbeitung der so entstehenden oder verschärften Konflikte beitragen kann.

\subsection{Großmachtbeziehungen/Machtverschiebungen}

Während die Transnationalismusforschung zu Recht ein wichtiges, wachsendes Phänomen der Internationalen Beziehungen heraushebt, sind vorzeitige Schwanengesänge auf das Abdanken des Nationalstaates unangebracht. Die Globalisierung fördert im Gegenteil selektiv das Erstarken vormals eher schwacher Entwicklungsstaaten. China, Indien und Brasilien stehen dabei in der ersten Reihe. Damit ergibt sich einmal mehr die bekannte Konstellation aufsteigender versus (relativ) absteigender Mächte. Anstatt einem durch nichts außer kindlichem Optimismus gestützten Befund, der große internationale Krieg sei auf dem Aschehaufen der Geschichte gelandet, gilt es, diese Konstellation, ihre Dynamik und ihre Konflikthaftigkeit auf das Sorgfältigste zu analysieren und Strategien zu entwickeln, um das Konfliktpotential abzufedern und einzuhegen. Dies betrifft nicht nur die Abwehr eines immensen Gemeinübels (»common bad«), nämlich einer globalen und womöglich nuklearen bewaffneten Auseinandersetzung, sondern es geht auch darum, durch die Minderung des Sicherheitsdilemmas Kooperationsbeziehungen mit dem Zweck der Schaffung von Gemeingütern, also Governance-Strukturen, möglich zu machen (Müller 2008). 


\subsection{Ethnologische Friedensforschung}

Die genannten drei Gebiete liegen solide in der Schnittmenge von Friedensforschung und IB. Als viertes Desiderat möchte ich - ganz aus der Sicht der Interdisziplinarität der Friedensforschung - kein Thema benennen, sondern die stärkere Einbindung einer Disziplin in die Friedensforschung, nämlich der Ethnologie. Dafür gibt es vier Gründe: Erstens macht das quantitative Überwiegen intragesellschaftlicher Konflikte im globalen Gewaltgeschehen das Verständnis des lokalen Gewaltpotentials höchst dringlich. Zweitens verlangt die Frage nach den ideationalen Grundlagen internationaler Politik, beispielsweise der Gerechtigkeitsproblematik, die Kenntnis kultureller Differenzen. Drittens führt das wachsende Interesse am Transnationalismus zwangsläufig dazu, dass der Blick auf das Phänomen der »Glokalisierung« fällt. $\mathrm{Zu}$ deren Aufhellung muss man auch wissen, was eigentlich im lokalen Bereich geschieht. Viertens ist der Schatz der enorm vielfältigen und diversen Methoden der Konfliktbearbeitung, die an allen möglichen Stellen der Welt entwickelt worden sind, noch lange nicht gehoben; die »Friedensstiftungsdiversität« ist wie die Biodiversität eine ebenso wertvolle wie bedrohte Grundlage menschlichen Zusammenlebens. Auch kommt man nicht ohne Ethnologie aus, um das Phänomen lokaler Friedensstiftung zu vermessen und zu katalogisieren. Die Ethnologie hat in ihren noch relativ seltenen Ausflügen in die Friedens- und Konfliktforschung ihr Potential längst erwiesen (vgl. Elwert et al. 1999; Schröter 2006). Es verspricht großen Nutzen, diese Disziplin stärker für die Friedensforschung nutzbar zu machen. Mithilfe einer Förderung durch die Leibniz-Gemeinschaft wird die HSFK hierzu eine Anstrengung unternehmen.

\subsection{Politische Ökonomie des Friedens}

Die Verschiebungen in der Weltwirtschaft, vor allem aber die Verwerfungen der globalen Finanzkrise, haben das Verhältnis zwischen wirtschaftlichen Entwicklungen und gewaltsamen Konflikten erneut in den Fokus gerückt. Aus den Augen verloren hat die Friedensforschung diesen Aspekt nie. So hat in den letzten beiden Jahrzehnten das innere Gewaltpotenzial von Rentenökonomien erhebliche Aufmerksamkeit gefunden und zu belastbaren Ergebnissen geführt, nicht zuletzt in den Arbeiten des Symposiumsteilnehmers Klaus Schlichte. Ähnliches gilt für das Konzept der Gewaltökonomien. Augenblicklich zeichnet sich eine noch weitaus stärker internationalisierte Problematik ab, die zum einen mit der des Aufstiegs neuer Mächte, zum anderen aber mit allgemeinen Gleichgewichts-, Stabilitäts- und Gerechtigkeitsproblemen der Weltwirtschaft verknüpft sind und erhebliche Fragen nach der Friedensfähigkeit der weltwirtschaftlichen Institutionen aufwerfen. 


\subsection{Politische Ökologie des Friedens}

Der Zusammenhang zwischen der Zerstörung der natürlichen Umwelt und inneren sowie internationalen Konflikten ist alles andere als neu. ${ }^{4} \mathrm{Er}$ ist indes durch die kontinuierlichen Entwicklungen zum Schlechteren und in den letzten beiden Jahrzehnten durch die immer evidentere Tatsache der globalen Erwärmung noch weitaus virulenter geworden, als er vor einer Generation war. Die Schere zwischen der Problementwicklung und einer von kurzfristigen Wirtschaftlichkeitserwägungen gehinderten problemadäquaten Bewältigungsstrategie nimmt allmählich so dramatische Folgen an, dass auch größere politische Verwerfungen in Folge der Erwärmungsfolgen wahrscheinlicher werden. Diese Problematik verdient intensive Aufmerksamkeit durch die Friedensforschung; das ISFH ist hier im Verbund des Hamburger Exzellenzclusters »Integrated Climate System Analysis and Prediction« (CliSAP) vorbildlich voran gegangen.

\section{Schluss: Kein Kampf der Kulturen}

Friedensforschung und IB kommen bestens miteinander aus. Das Verhältnis deckt die Aspekte von Teilidentität, Schnittmenge, Synergie und Kooperation ab, je nachdem, welchen Aspekt man behandelt. Die wesentlichen Unterschiede - Interdisziplinarität und klare normative Vorgaben sowie praxeologische Verpflichtungen der Friedensforschung, Disziplinarität mit verschwimmenden Grenzen und explizite normative und praktische Orientierung als Option auf Seiten der IB - sind zu gering, um Entfremdung oder gar Konflikt zu begründen: Der Kampf der Kulturen findet jedenfalls zwischen Friedensforschung und IB nicht statt. Auch in den inhaltlichen Desideraten gibt es eine wichtige Überlappung, wenngleich die IB jenseits der hier vorgeschlagenen Prioritäten natürlich noch eine größere eigene Agenda besitzt. Abschließend lässt sich sagen: Inmitten aller Konflikte und ideologischen Streitigkeiten herrscht über diesen Gipfeln eine angenehme Ruhe.

\section{Literatur}

Albin, Cecilia 2001: Justice and Fairness in International Negotiation, Cambridge.

Baumgart-Ochse, Claudia/Schörnig, Niklas/Wisotzki, Simone/Wolff, Jonas (Hrsg.) 2011: Auf dem Weg zu Just Peace Governance. Beiträge zum Auftakt des neuen Forschungsprogramms der HSFK, Baden-Baden.

Booth, Ken 2007: Theory of World Security, Cambridge.

Czempiel, Ernst Otto 1998: Friedensstrategien. Eine systematische Darstellung außenpolitischer Theorien von Machiavelli bis Madariaga, Opladen.

4 Der Autor selbst hat in unvordenklichen Zeiten einen der ersten deutschen Beiträge zu dieser Thematik abgeliefert (Müller 1980). 
Elwert, Georg/Feuchtwang, Stephan/Neubert, Dieter (Hrsg.) 1999: The dynamics of violence. Processes of escalation and de-escalation in violent group conflicts, Berlin.

Galtung, Johan 1997: Preis der Modernisierung. Struktur und Kultur im Weltsystem, Wien.

Gray, Colin S./Payne, Keith 1980: Victory is Possible, in: Foreign Policy 9: 2, 14-27.

Hellmann, Gunther/Müller, Harald 2004: Einleitung: Zehn Jahre Zeitschrift für Internationale Beziehungen. Was wir aus den Geburtstagsgrüßen lernen, in: Zeitschrift für Internationale Beziehungen 11:2, 273-280.

Krause, Keith/Williams, Michael C. 1997: Critical Security Studies: Concepts and Cases, London.

Mayer, Peter 2006: Macht, Gerechtigkeit und internationale Kooperation. Eine regimeanalytische Untersuchung zur internationalen Rohstoffpolitik, Baden-Baden.

Morgenthau, Hans J. 1948: Politics Among Nations: The Struggle for Power and Peace, New York, NY.

Müller, Harald 1980: Wachstumskrise und Friedensforschung, in: Hessische Stiftung Friedens- und Konfliktforschung (Hrsg.): Friedensanalysen 11, Frankfurt a. M., 141-177.

Müller, Harald 2003: Begriff, Theorien und Praxis des Friedens, in: Hellmann, Gunther/Wolf, Klaus Dieter/Zürn, Michael (Hrsg.): Die neuen Internationalen Beziehungen. Forschungsstand und Perspektiven in Deutschland, Baden-Baden, 209-250.

Müller, Harald 2006: Politikberatung in unterschiedlichen Kontexten: Notizen aus der Praxis, in: Hellmann, Gunther (Hrsg.): Forschung und Beratung in der Wissensgesellschaft. Das Feld der internationalen Beziehungen und der Außenpolitik, Baden-Baden, 213-248.

Müller, Harald 2008: Wie kann eine neue Weltordnung aussehen? Wege in eine nachhaltige Politik, Frankfurt a. M.

Müller, Harald 2011: Demokratie, Gerechtigkeit und Frieden: Die guten Dinge gehen nicht immer zusammen, in: Baumgart-Ochse et al. 2011, 277-309.

Rat der Europäischen Union 2010: Beschluss des Rates zur Schaffung eines Europäischen Netzes unabhängiger Reflexionsgruppen für Nichtverbreitungsfragen zur Unterstützung der Umsetzung der Strategie der EU gegen die Verbreitung von Massenvernichtungswaffen, 5382/10, Brüssel, 7.7.2010.

Schröter, Susanne 2006: Politisierung von Religion und Sakralisierung von Politik. Lokale und nationale Konflikte zwischen Moslems und Christen in Indonesien, in: Oevermann, Ulrich/Franzmann, Manuel (Hrsg.): Religiosität in der säkularisierten Welt. Theoretische und empirische Beiträge zur Säkularisierungsdebatte in der Religionssoziologie, Wiesbaden, 357-374.

Senghaas, Dieter 1971: Kritische Friedensforschung, Frankfurt a. M.

Smith, Tony 2007: A Pact With the Devil: Washington's Bid for World Supremacy and the Betrayal of the American Promise, New York, NY.

Welch, David 1993: Justice and the Genesis of War, Cambridge. 